\title{
Isoform-specific palmitoylation of JNK regulates axonal development
}

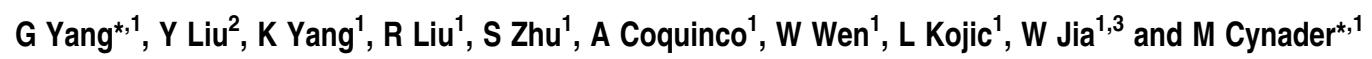

The c-jun N-terminal kinase (JNK) proteins are encoded by three genes (Jnk1-3), giving rise to 10 isoforms in the mammalian brain. The differential roles of JNK isoforms in neuronal cell death and development have been noticed in several pathological and physiological contexts. However, the mechanisms underlying the regulation of different JNK isoforms to fulfill their specific roles are poorly understood. Here, we report an isoform-specific regulation of JNK3 by palmitoylation, a posttranslational modification, and the involvement of JNK3 palmitoylation in axonal development and morphogenesis. Two cysteine residues at the COOH-terminus of JNK3 are required for dynamic palmitoylation, which regulates JNK3's distribution on the actin cytoskeleton. Expression of palmitoylation-deficient JNK3 increases axonal branching and the motility of axonal filopodia in cultured hippocampal neurons. The Wnt family member Wnt7a, a known modulator of axonal branching and remodelling, regulates the palmitoylation and distribution of JNK3. Palmitoylation-deficient JNK3 mimics the effect of Wnt7a application on axonal branching, whereas constitutively palmitoylated JNK3 results in reduced axonal branches and blocked Wnt7a induction. Our results demonstrate that protein palmitoylation is a novel mechanism for isoform-specific regulation of JNK3 and suggests a potential role of JNK3 palmitoylation in modulating axonal branching.

Cell Death and Differentiation (2012) 19, 553-561; doi:10.1038/cdd.2011.124; published online 23 September 2011

The c-jun N-terminal kinase (JNK) family consists of JNK1, JNK2 and JNK3 subgroups, which participate in diverse biological and pathological processes. ${ }^{1,2}$ At least $10 \mathrm{JNK}$ isoforms of varied sizes, with most between 46 and $54 \mathrm{kDa}$, are produced from the Jnk1-3 genes, giving rise to JNK isoforms. ${ }^{1}$ In the nervous system, JNKs (particularly isoform JNK3) have been extensively studied as the key players in apoptosis and neurodegeneration. ${ }^{3-6}$ However, accumulating evidence supports a physiological role of JNK in regulating neurite formation and morphogenesis. ${ }^{7-12}$ Pharmacological inhibition of JNK activity blocks axogenesis in hippocampal neurons, arguing for an essential role of JNK in neurite development. ${ }^{13}$ Growth factors and signalling molecules, including secreted proteins of the Wnt family, have also been found to activate JNK for remodelling dendrites and axons, a process that relies on cytoskeletal rearrangement. ${ }^{11,14-16}$ This has led to the identification of several actin- or microtubule-associated proteins as JNK substrates that modulate different aspects of cytoskeletal activity. ${ }^{17}$ However, all the $10 \mathrm{JNK}$ isoforms share the same kinase domain and activation mechanism. ${ }^{17}$ The differential roles of JNK isoforms in neurite development and the mechanisms underlying isoform-specific regulation are poorly understood.

Analysis of animals null for particular JNK isoforms provides the first evidence to support isoform-specific roles of JNKs in the brain. Mice with $J n k 1^{-/-}$show abnormalities in neurite development, ${ }^{7}$ whereas mice null for Jnk1 and Jnk2 show embryonic lethality due to severe neurological defects. ${ }^{18}$ Jnk $2^{-/-}$mice, but not $J n k 1^{-1-}$ mice, also show enhanced protection against brain damage in Parkinson's disease models. ${ }^{4} \mathrm{JNK} 1$ and JNK2 are thus implicated in regulating brain development, as well as neuronal death. In contrast, Jnk $3^{-1-}$ mice apparently develop normally, but show enhanced resistance to stress-induced neurodegeneration. ${ }^{3-5} \mathrm{JNK} 3$ is thus considered as a key regulator of neuronal cell death, rather than that of neuronal development. However, recent studies highlight a physiological role of JNK3 in neuronal differentiation and neurite growth, ${ }^{10,12}$ raising the possibility that each isoform may be differentially regulated to achieve selective functions. An understanding of how JNK isoforms are specifically regulated is essential for elucidating JNK isoform-specific roles in multiple biological processes.

Isoform-selective regulation has been observed in some JNK scaffolding proteins that regulate JNK activity and trafficking. ${ }^{1}$ Another potential mechanism may be posttranslational modification. In addition to phosphorylation that activates JNKs, and S-nitrosylation that inhibits JNK activity, little is known about whether JNKs are subjected to other modifications. ${ }^{2,19}$ Protein palmitoylation is one such candidate for modification. It dynamically regulates protein trafficking

\footnotetext{
${ }^{1}$ College of Interdisciplinary Studies, University of British Columbia, Brain Research Centre, 2211 Wesbrook Mall, Vancouver, BC V6T2B5, Canada; ${ }^{2}$ Department of Pathology, School of Preclinical Medicine, Beijing University of Chinese Medicine, 11 Bei San Huan Dong Road, Chaoyang District, Beijing, 100029, China and ${ }^{3}$ Department of Surgery, University of British Columbia, 2211 Wesbrook Mall, Vancouver, BC V6T2B5, Canada

*Corresponding authors: G Yang or M Cynader, College of Interdisciplinary Studies, University of British Columbia, Brain Research Centre, 2211 Wesbrook Mall, Vancouver, BC V6T2B5, Canada. Tel: + 1604822 1388; Fax: + 1604822 0361; E-mail: photonyg@interchange.ubc.ca (GY) or cynader@brain.ubc.ca (MC)

Keywords: c-Jun N-terminal kinase/JNK; palmitoylation; axonal branching; isoform regulation; cytoskeleton; Wnt pathway

Abbreviations: JNK, c-jun N-terminal kinase; PAT, palmitoyl acyl transferase; 2-BrPA, 2-bromopalmitate; HAM, hydroxylamine; GFP, green fluorescence protein; zD15/20/23, zinc-finger DHHC containing 15/20/23; MI, motility index; Cyt. D, cytochalasin D; Lat. A, latrunculin A; TR-phalloidin, Texas Red-phalloidin; PSD-95, postsynaptic protein 95; MAP2, microtubule associated protein 2

Received 20.5.11; revised 12.8.11; accepted 20.8.11; Edited by N Bazan; published online 23.9.11
} 
and functions by reversibly attaching the lipid palmitate to cysteine (Cys) residues. ${ }^{20,21}$ This modification is catalyzed by the family of palmitoyl acyl transferases (PATs) containing 24 members, and is sensitive to protein sequence and structure. ${ }^{20}$ Proteins with little sequence variance may differ in palmitoylation sensitivity and specificity, ${ }^{21-23}$ such as glutamate receptor interacting protein 1 isoform $a$ and $b,{ }^{23}$ providing a potential way to achieve isoform-specific regulation. Sequence alignment of JNK isoforms indicates that differences exist at the $\mathrm{NH}_{2}$ - and $\mathrm{COOH}$-termini, potential regions for isoform-specific regulation (Supplementary Figure S1). Indeed, the $\mathrm{NH}_{2}$-terminus of JNK3 has been shown to selectively interact with the scaffolding protein $\beta$ arrestin2. ${ }^{24}$ The Cys residues that are likely targets for palmitoylation are located at the $\mathrm{COOH}$-termini of JNK3 and are not found on the p46 isoforms.

In this study, we demonstrate that JNK3 is the predominant isoform that is palmitoylated in neurons. With this isoformspecific regulation, JNK3 is found to have a role in axon branching and filopodia motility, possibly by modulating cytoskeletal components, as palmitoylation affects JNK3 trafficking to the triton-insoluble actin cytoskeletal fraction. This palmitoylation-regulated function of JNK3 responds to Wnt signals and is necessary for Wnt7a-induced axonal branching. Our results demonstrate that palmitoylation is a JNK isoform-specific regulation and suggest that the rapid and dynamic palmitoylation on JNK3 has an essential role in axonal growth and branching during development.

\section{Results}

Palmitoylation occurs primarily on a particular JNK3 isoform. After metabolic labelling of cortical neuronal cultures, we detected ${ }^{3} \mathrm{H}$-palmitate incorporation into JNK3, which was eliminated by co-incubation with the palmitoylation inhibitor 2-bromopalmitate (2-BrPA) (Figure 1a). In vitro application of hydroxylamine (HAM), which breaks covalent bonds and releases ${ }^{3} \mathrm{H}$-palmitate from the protein, also abolished the signal, confirming that JNK3 is a palmitoylated protein in neurons. A pulse-chase strategy was used to examine the kinetics of palmitoylation on JNK3. We found a highly dynamic cycling of palmitate on JNK3 with a calculated half-life of about $4 \mathrm{~h}$ in neurons and in the HEK293 cells expressing GFP-JNK3 (Figure 1b, Supplementary Figure S2). To test if JNK3 shows a preference for certain PATs, several neuronal PATs (zD15, zD20 and zD23) with different expression levels in the brain were expressed individually with GFP or GFP-tagged JNK3 in heterologous HEK293 cells. PATs zD15 and zD20 significantly promoted JNK3 palmitoylation (Figure 1c). However, introducing zD23 or a zD15 mutant without PAT activity (zD15 $\Delta$ ) failed to enhance JNK3 palmitoylation (Figures $1 \mathrm{c}$ and $\mathrm{d}$ ), suggesting that JNK3 may be the substrate of a selective group of PATs in neurons. Among the JNK isoforms with an extended $\mathrm{COOH}-$ terminus, JNK1 and JNK3, but not JNK2, have two Cys residues located at the end of the terminus (Figure $1 \mathrm{~d}$ ). ${ }^{1}$ We next investigated whether these Cys residues are potential

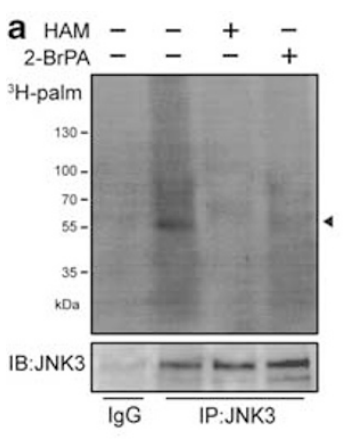

d

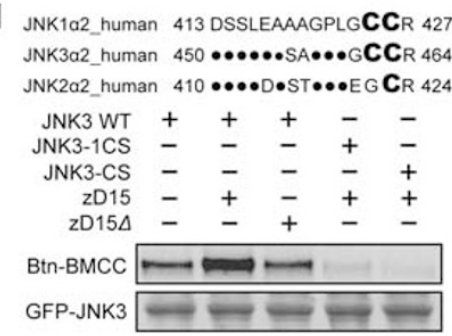

b

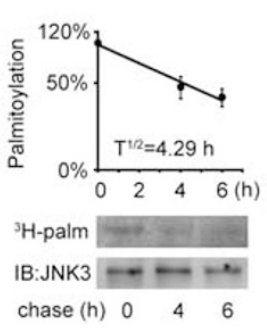

C

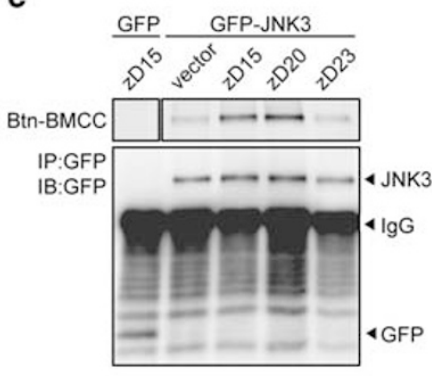

e

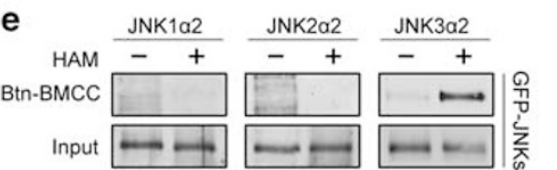

Figure 1 JNK3 is palmitoylated at the $\mathrm{COOH}$-terminus. (a) Metabolic labelling shows that JNK3 is palmitoylated in cortical neurons. JNK3 palmitoylation was examined by metabolic labelling with ${ }^{3} \mathrm{H}$-palmitic acid $\left({ }^{3} \mathrm{H}\right.$-palm) (see Materials and Methods). Pre-treating neurons with 2-BrPA or adding HAM to JNK3 immunoprecipitates eliminated metabolic labelling signals. Normal rabbit IgG served as the immunoprecipitation control. The arrowhead indicates JNK3. (b) Palmitate on JNK3 has a short half-life. Pulsechase experiments (chased at $0,4,6 \mathrm{~h}$ ) show a short half-life (calculated $\sim 4.3 \mathrm{~h}, n=5$ ) of palmitate on endogenous JNK3 in cortical neurons. (c) PATs show selectivity in palmitoylating JNK3 in heterogeneous HEK293 cells. PAT zD15 and zD20, but not zD23, enhances JNK3 palmitoylation, assessed by Btn-BMCC labelling (see Materials and Methods). (d) Two Cys residues at the $\mathrm{COOH}$-terminus of JNK3 are critical for palmitoylation. The sequence alignment of the last 15 amino acids at the $\mathrm{COOH}$-termini of JNK p54 isoforms reveals potential Cys residues (bold letters) for palmitoylation. Point-mutation of one or both Cys residues to Ser eliminates JNK3 palmitoylation, in the presence of PAT zD15. PAT activity-deficient zD15 (zD15 $\Delta$ ) loses the ability to promote JNK3 palmitoylation. (e) Palmitoylation occurs predominantly on the JNK3 isoform. The palmitoylation status of JNK p54 isoforms JNK1 $\alpha 2$, JNK2 22 and JNK3 $\alpha 2$, overexpressed in HEK293 cells, assessed by Btn-BMCC labelling, are shown and compared with the background (HAM minus). The schematic diagram at the bottom shows that JNK3 is the major isoform that is palmitoylated 
sites for JNK3 palmitoylation by replacing Cys with Ser. In the presence of PAT zD15, loss of one Cys was enough to abolish JNK3 palmitoylation in HEK293 cells, indicating that both Cys residues of JNK3 are required for normal palmitoylation. This implies that JNK2 with only one Cys residue may not undergo palmitoylation. Indeed, although JNK3 palmitoylation was clearly detected, we were unable to detect JNK2 p54 palmitoylation above the background control, assessed in heterologous cells (Figure 1e). To our surprise, we also found that JNK1 p54 was not a palmitoylated protein though it has two Cys residues at the $\mathrm{COOH}$-terminus (Figure 1e). This point is considered further in the discussion section. Thus, JNK3 is the major isoform that is palmitoylated in neurons.

Axonal branching and filopodia motility are regulated by JNK3 palmitoylation. We first suspected that palmitoylation may affect JNK3 phosphorylation under stress conditions. However, similar phosphorylation levels of JNK3 wild type (JNK3 WT) and palmitoylation-deficient JNK3 mutant (JNK3 CS) were observed in heterologous cells challenged with or without osmotic stress (Supplementary Figure S3), implying that palmitoylation may not be involved in the JNK3regulated stress response. Recent studies have suggested a physiological role of JNK3 in neuronal differentiation and neurite growth. ${ }^{10,12}$ To study the potential role played by JNK3 palmitoylation in axonal development, we transfected cultured hippocampal neurons with the indicated constructs together with DsRed2 at 5 days in vitro (DIV) for $48 \mathrm{~h}$. DsRed2 expression filled the neuron and was used to track the morphological changes of axons. At 7 DIV, neurons generated long and branched axons that were clearly labelled with both DsRed2 and the axonal marker Tau-1, and several short dendrites around neuronal somata, where only DsRed2 signals were detected (Figure 2a). Axons from neurons overexpressing JNK3 WT showed similar structures to those of the DsRed2 control. In contrast, overexpressing JNK3 CS significantly promoted axonal complexity, with a marked increase in branch numbers (Figure 2b, Supplementary Table S1). We quantified axonal branching by measuring the axonal branch number of different branching orders. ${ }^{25}$ Neurons overexpressing JNK3 CS showed more branches at all branching orders (secondary, $6.5 \pm 0.3, \quad P<0.01$; tertiary, $2.7 \pm 0.2, \quad P<0.01$; higher, $0.4 \pm 0.1, P<0.01$ ) and doubled the total branch number, compared with neurons with DsRed2 alone (secondary, $3.3 \pm 0.2$; tertiary, $0.7 \pm 0.1$; higher, $0.0 \pm 0.0$ ) or JNK3 WT (secondary, $3.8 \pm 0.2$; tertiary, $0.8 \pm 0.2$; higher, $0.0 \pm 0.0$ ) (Figure 2c). Moreover, compared with controls, JNK3 CS induced a significant increase in total branch length (Figure 2c), although the length of the primary axon decreased. To test whether enhanced axonal branching was due to increased formation of filopodia, we assessed the density of axonal filopodia. As shown in Figure $2 \mathrm{~d}$, filopodial density measured in neurons overexpressing JNK3 CS did not differ from that in controls. However, time-lapse recording showed that axonal filopodia were more dynamic on extension and retraction in JNK3 CS neurons (Figure 2e). To directly visualize the dynamics of filopodia, we adopted a protocol to sum up intensity differences of each pixel between successive frames during the period of recording. ${ }^{26}$ Compared with controls, axonal filopodia in neurons overexpressing JNK3 CS were shown to display higher motility (Figure 2e), which was further quantified with a motility index (MI). ${ }^{26}$

Palmitoylation regulates JNK3 trafficking to cytoskeleton. Palmitoylation is an essential signal for facilitating protein association with lipid rafts, which act as platforms for signal transduction. JNK has been shown to be recruited to lipid rafts, where it can initiate downstream pathways. ${ }^{27}$ We thus examined the role of palmitoylation in lipid raft targeting of JNK3. Lipid rafts are plasma membrane micro-domains rich in cholesterol, which resist extraction with detergents. ${ }^{28}$ To isolate lipid rafts, cell lysates treated with Triton X-100 were subjected to Optiprep density-gradient centrifugation. ${ }^{28}$ The lipid raft marker caveolin-1 showed an intensity peak at fractions 8 and 9 (Figure $3 a$, left panel). Treating cells with methyl- $\beta$-cyclodextrin, a drug known for cholesterol depletion and lipid raft destruction, ${ }^{28}$ reduced the presence of caveolin-1 at fractions 8 and 9 , suggesting the enrichment of lipid rafts in these fractions. The distribution of JNK3 WT and JNK3 CS in fractions 8 and 9 showed no significant difference (Figure $3 a$, right panel). Similar results were found with JNK3 WT co-expressed with PAT zD15 to promote JNK3 palmitoylation. Moreover, unpalmitoylated JNK2 p54 was also present in lipid rafts. Hence, palmitoylation does not regulate the lipid raft association of JNK3. Nevertheless, compared with JNK3 WT, an increase of JNK3 CS and a small decrease of JNK3 WT co-expressed with zD15 were detected at fraction 13, the high-density triton-insoluble fraction enriched with actinassociated cytoskeleton. ${ }^{29}$ This implies that palmitoylation may affect JNK3's association with the actin cytoskeleton. Signals for actin cytoskeleton modulation are essential for axonal development. ${ }^{30}$ To analyze this quantitatively, ${ }^{29}$ we isolated the Triton-insoluble actin cytoskeleton fraction from cortical neurons and HEK293 cells expressing GFP-JNK3, followed by detection of JNK3. Consistently, JNK3 was detected in the Triton-insoluble fraction, which was sensitive to the actin depolymerizers cytochalasin $D$ (Cyt.D) or latrunculin A (Lat.A) (Figure 3b). JNK3 expressed in HEK293 cells was also found to be present in the actin depolymerizer-sensitive Triton-insoluble fraction (data not shown). We next investigated whether palmitoylation affected JNK3's association with the actin cytoskeleton. JNK3 constructs were expressed in HEK293 cells, and a twofold increase of JNK3 CS was detected in the Triton-insoluble fraction, compared with JNK3 WT (Figure 3c). Consistently, enhancing the JNK3 palmitoylation by co-expressing PAT zD15 caused a $50 \%$ reduction of JNK3 in the Triton-insoluble fraction.

Wnt7a-induced axonal branching is modulated by JNK3 palmitoylation. The Wnt family member Wnt7a is expressed in the hippocampal formation. ${ }^{31}$ It has been shown to modulate axonal development ${ }^{15}$ and to activate the non-canonical JNK pathway, ${ }^{16}$ raising the possibility that JNK3 palmitoylation may have a role in Wnt7a-induced axonal branching. We incubated five DIV hippocampal 
a

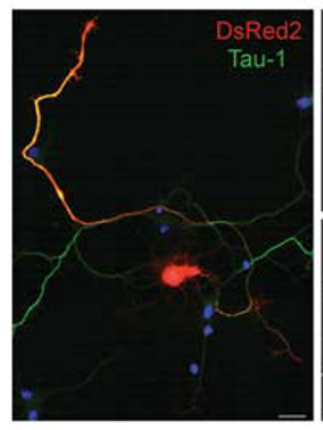

c

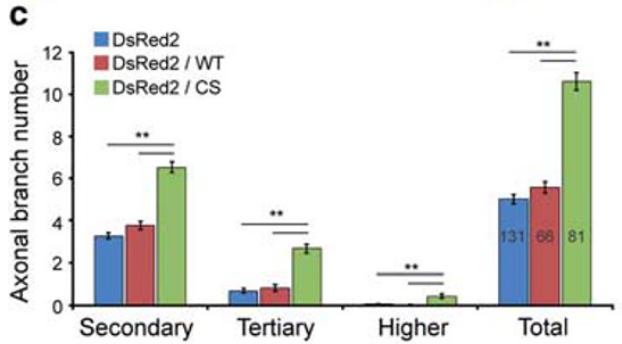

e

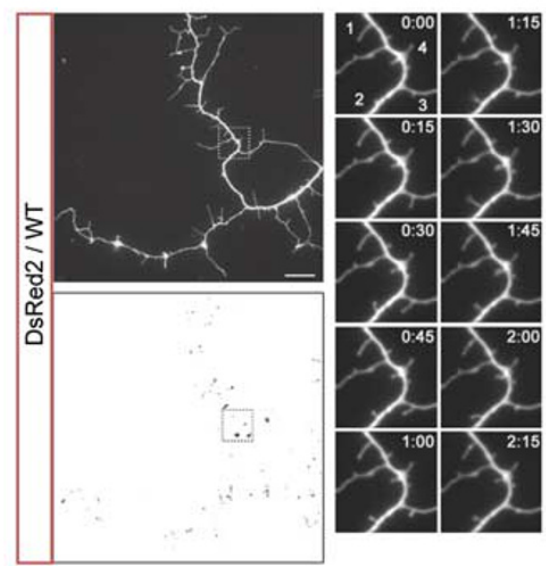

$\mathrm{MI}=0.40 \pm 0.02, n=200$ filopodia

b

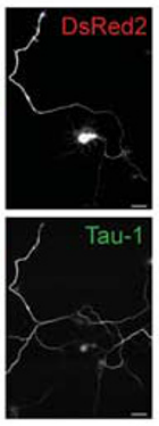

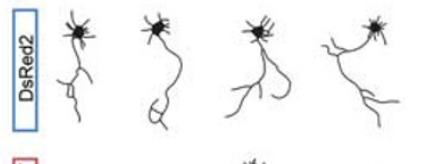
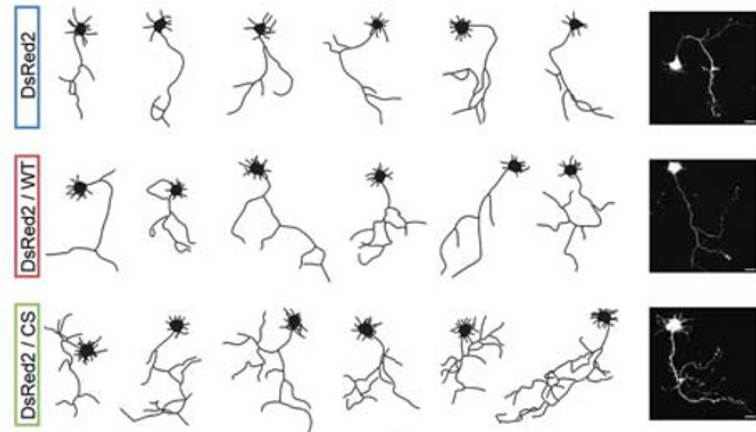

,
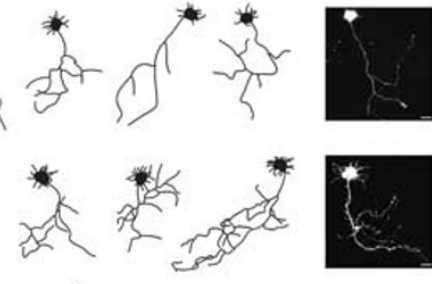

d
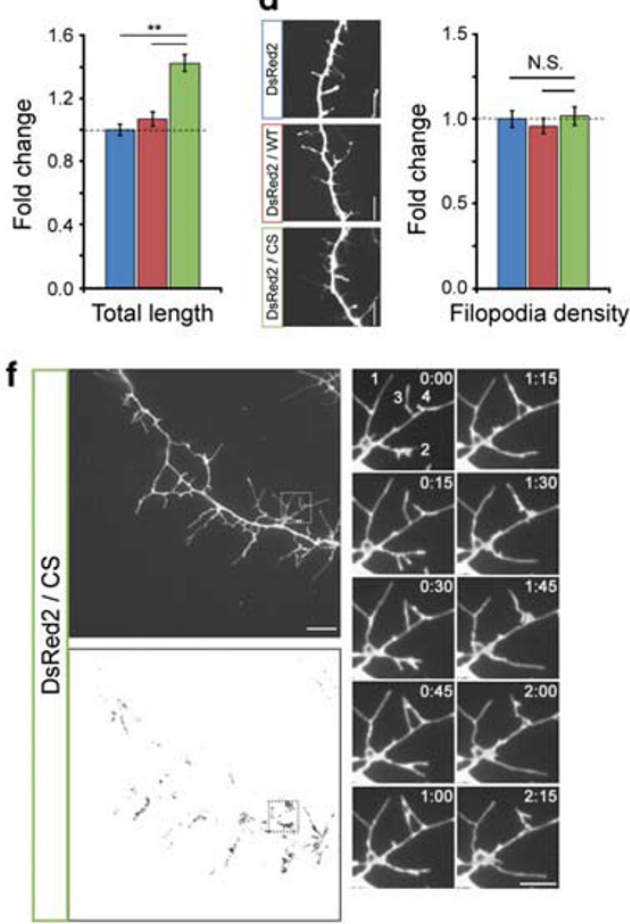

$\mathrm{MI}=0.70 \pm 0.03, \mathrm{n}=200$ filopodia

Figure 2 The palmitoylation-deficient JNK3 mutant promotes axonal branching and filopodia motility. (a) Long and branched axons are observed in cultured hippocampal neurons at 7 DIV. Axons are labelled with the axonal marker Tau-1 (green) and DsRed2. Dendrites that are only labelled with DsRed2 are short and located near the somata. Scale bar: $10 \mu \mathrm{m}$. (b) Overexpression of the palmitoylation-deficient JNK3 mutant (JNK3 CS) increases axon length and branch complexity. Representative neurons transfected with DsRed2 alone, or together with JNK3 wild type (WT) or JNK3 CS, are shown. Scale bar: $10 \mu \mathrm{m}$. (c) Axonal branch numbers and total length of axons are enhanced by JNK3 CS. The tip numbers of secondary, tertiary and higher order of branches are shown. JNK3 CS increases the total branch number of axons (DsRed2 alone/ blue, $5.0 \pm 0.2, n=131$; WT/red, $5.6 \pm 0.3, n=66$; CS/green, $10.6 \pm 0.4, n=81$ ) and total axonal length (fold change to DsRed2 alone, WT, $1.1 \pm 0.1 ;$ CS, 1.4 \pm 0.1 ). Quantifications show mean \pm S.E.M. $t$-test. ${ }^{* *} P<0.01$. (d) The density of axonal filopodia is not affected by JNK3 CS. Representative figures show axonal filopodia from transfected neurons. Scale bar: $5 \mu \mathrm{m}$. No significant changes in the CS group are observed (fold change to DsRed2 alone, WT, 1.0 $\pm 0.1 ; C S, 1.0 \pm 0.1, P=0.85$ to DsRed2 alone, $P=0.40$ to WT). (e) Axonal filopodia motility is promoted by JNK3 CS. Representative figures show the motility of axonal filopodia in WT and CS groups. The motility of axonal filopodia is further transformed into a single image and is shown below. Scale bar: $5 \mu \mathrm{m}$. Regions of marked dotted-squares are magnified and time-lapse images are shown with indicated time points within $135 \mathrm{~s}$. Four labelled filopodia, as selected examples in the CS group, are more mobile than those in the WT group. Scale bar: $5 \mu \mathrm{m}$. The motility of axonal filopodia (10 filopodia per neuron from 20 neurons) is quantified with the MI (DsRed alone, $0.4 \pm 0.0, n=200 ;$;T, $0.4 \pm 0.0, n=200, P=0.12$; CS, $0.7 \pm 0.0, n=200, P<0.01)$

neurons with recombinant Wnt7a for $6 \mathrm{~h}$ and assessed endogenous JNK3 palmitoylation. ${ }^{32,33}$ Wnt7a treatment markedly reduced neuronal JNK3 palmitoylation to $50 \%$ of the untreated control, whereas the palmitoylation status of the total protein complements showed no obvious change (Figure 4a). The Wnt7a treatment also caused endogenous JNK3 translocation to the Triton-insoluble cytoskeleton fraction (Figure 4b). Incubation with Wnt7a for $48 \mathrm{~h}$ promoted axonal branching in neurons overexpressing JNK3 WT (secondary, $5.4 \pm 0.3$; tertiary, $2.4 \pm 0.3$; higher orders, $0.2 \pm 0.1$; total, $9.5 \pm 0.7$ ) or DsRed2 alone (secondary, 5.4 \pm 0.3 ; tertiary, $2.5 \pm 0.3$; higher orders, $0.2 \pm 0.1$; total, $9.1 \pm 0.5)$, compared with the untreated control (secondary, $3.5 \pm 0.3, P<0.01$; tertiary, $0.5 \pm 0.1$, 
a
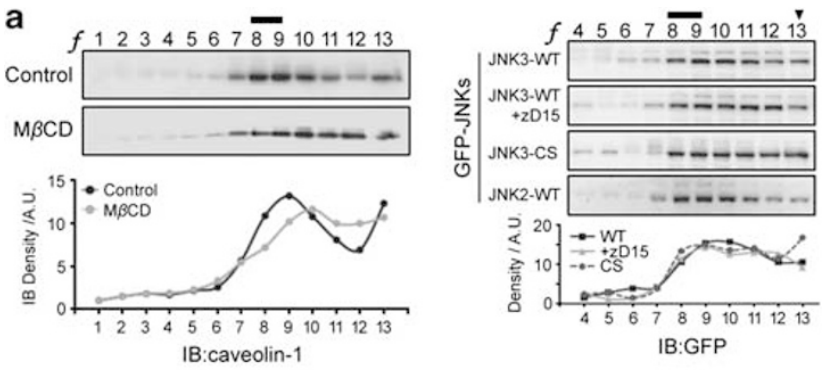

b
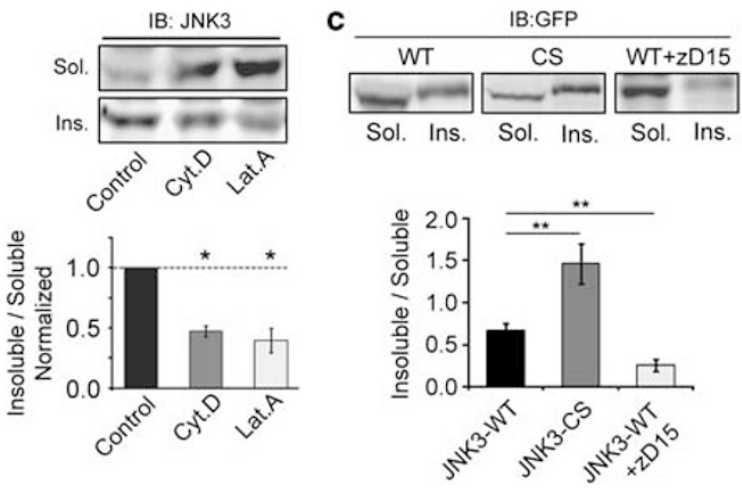

Figure 3 Palmitoylation regulates the translocation of JNK3 to the Tritoninsoluble actin cytoskeleton. (a) Palmitoylation does not affect JNK3 trafficking to lipid rafts. Lysates of HEK293 cells expressing the indicated constructs are subjected to density fractionation (see Materials and Methods). Fractions from Optiprep density-gradient centrfugation (f 1-13) are shown. Quantifications of the immunoblot intensity of the lipid raft marker caveolin-1 or JNKs from each fraction are shown at the bottom. Fractions 8 and $9(f 8,9)$ are marked as lipid rafts. Note the increase of JNK3 CS in fraction 13. (b) Endogenous neuronal JNK3 is present in the Triton X-100 insoluble-cytoskeleton fraction. In cortical neurons, incubation with Cyt.D or Lat.A reduces the presence of JNK3 in the insoluble fraction as calculated by the insoluble/soluble ratio (fold change to control, Cyt.D, $0.5 \pm 0.1$; Lat.A, $0.4 \pm 0.11$ ). Quantifications show mean \pm S.E.M. $t$-test. ${ }^{*} P<0.05$. Representative figures are from three independent experiments. (c), Palmitoylation regulates JNK3 translocation to the Triton X-100 insoluble fraction. The Triton X-100 solubility of JNK3 expressed in HEK293 was analyzed. The insoluble/soluble ratios of GFP-tagged JNK3 WT, JNK3 CS and JNK3 WT together with PAT zD15 are shown (WT, $0.7 \pm 0.1 ; \mathrm{CS}, 1.5 \pm 0.2$; WT $+\mathrm{zD} 15,0.3 \pm 0.1$ ). $t$-test. ${ }^{*} P<0.01$

$P<0.01$; higher, $0.0 \pm 0.0, P<0.05$; total, $5.0 \pm 0.4, P<0.01$ ) (Figures $4 \mathrm{c}$ and d, Supplementary Table S1). However, Wnt7a stimulation did not further enhance axonal branching in JNK3 CS-transfected neurons (secondary, 6.1 \pm 0.4 ; tertiary, $2.8 \pm 0.3$; higher, $0.4 \pm 0.2 ;$ total, $10.3 \pm 0.6$ ) compared with JNK3 CS-transfected neurons without Wnt7a treatment (Figures $2 \mathrm{c}$ and $4 \mathrm{c}, \mathrm{d}$ ). This suggests that overexpressing JNK3 CS stimulated the effect of Wnt7a on axonal branching and thus prevented any further enhancement when Wnt7A was administered. Next, we fused the prenylated and dual palmitoylated motif of paralemmin to the $\mathrm{COOH}$-terminus of JNK3 CS to mimic a constant pseudo-palmitoylation on JNK3 (JNK3 Parlm). ${ }^{34-36}$ The effects of Wnt7a on axonal branching were completely blocked in neurons overexpressing JNK3 Parlm (secondary, $2.8 \pm 0.2, \quad P<0.01$; tertiary, $0.5 \pm 0.1, \quad P<0.01$; higher, $0.0 \pm 0.0, P<0.01$; total, $4.3 \pm 0.3, P<0.01$ ) compared with WT treated with Wnt7a (Figures $4 \mathrm{c}$ and d), suggesting that it is in fact JNK3 palmitoylation that modulates Wnt7a-induced axonal branching. Furthermore, overexpression of JNK3 Parlm was found to reduce axonal branching and total axon length in untreated neurons, compared with controls (Figure 4e). Together, these results indicate that rapid and dynamic palmitoylation of JNK3 may be an essential regulator of axonal development.

\section{Discussion}

Although the identification of JNK isoform-specific substrates has greatly improved our understanding of mechanisms underlying the differential roles played by JNK isoforms, ${ }^{1,17}$ much remains unknown about how JNK isoforms themselves are differentially regulated. Our results demonstrate protein palmitoylation as a novel isoform-specific mechanism for JNK3 regulation, and reveal a role of JNK3 palmitoylation in axonal branching in response to Wnt signals.

Based on previous studies, ${ }^{1,24}$ we assumed that JNK isoform-specific regulation may occur at regions unique to certain isoforms. We compared protein sequences of JNK isoforms. In the brain, Jnk1 and Jnk2 predominantly produce p46 and p54 isoforms, respectively, whereas Jnk3 produces an isoform at $49 \mathrm{kDa}$ (Supplementary Figure S1). ${ }^{1,37}$ The $\mathrm{COOH}$-termini of the p54 isoforms of JNK2 and JNK3 are different from other isoforms, raising the possibility that this region may be manipulated by certain mechanisms for isoform-specific regulation. One and two Cys residues are found on JNK2 p54 and JNK3, respectively. We thus suspect that these isoforms may be modified by palmitoylation. By using metabolic labelling and point mutation analysis, we confirmed that JNK3 is a palmitoylated protein and both Cys residues are required for palmitoylation. In contrast, JNK2 p54, which contains only one Cys residue, is not palmitoylated. Interestingly, although JNK1 p54 has a $\mathrm{COOH}$-terminal sequence similar to that of JNK3, JNK1 failed to be palmitoylated in our experiments. It has been noticed that palmitoylation is sensitive to variation in protein sequence and structure. ${ }^{21,23}$ Small differences of amino acids at the $\mathrm{COOH}$-termini between JNK1 and JNK3, or different protein structures may account for their distinct palmitoylation status. However, we do not exclude the possibility that the JNK1 p54 isoform may be palmitoylated in cell types where JNK3 is absent or under certain circumstances. Indeed, a transition of roles for JNK isoforms has been observed in the brain after ischemic challenge. ${ }^{38}$ Because products of the Jnk1 gene in neurons are primarily p46 isoforms that lack extended $\mathrm{COOH}$-termini for palmitoylation, ${ }^{39}$ JNK3 is therefore the major isoform that is palmitoylated under our experimental conditions. This novel JNK3 palmitoylation provides evidence for the idea that certain JNK isoforms may undergo other posttranslational modifications in addition to the extensively studied phosphorylation.

JNK proteins are ubiquitously distributed within the cell. In response to internal and external stimuli initiated from diverse origins, JNK is activated and transduces signals by phosphorylating its substrates found at various loci within the cell, such as c-jun in the nucleus, MAP2 on microtubules, paxillin on the actin cytoskeleton, Bcl2 in mitochondria, and others. ${ }^{17}$ Given the simultaneous presence of JNK isoforms 

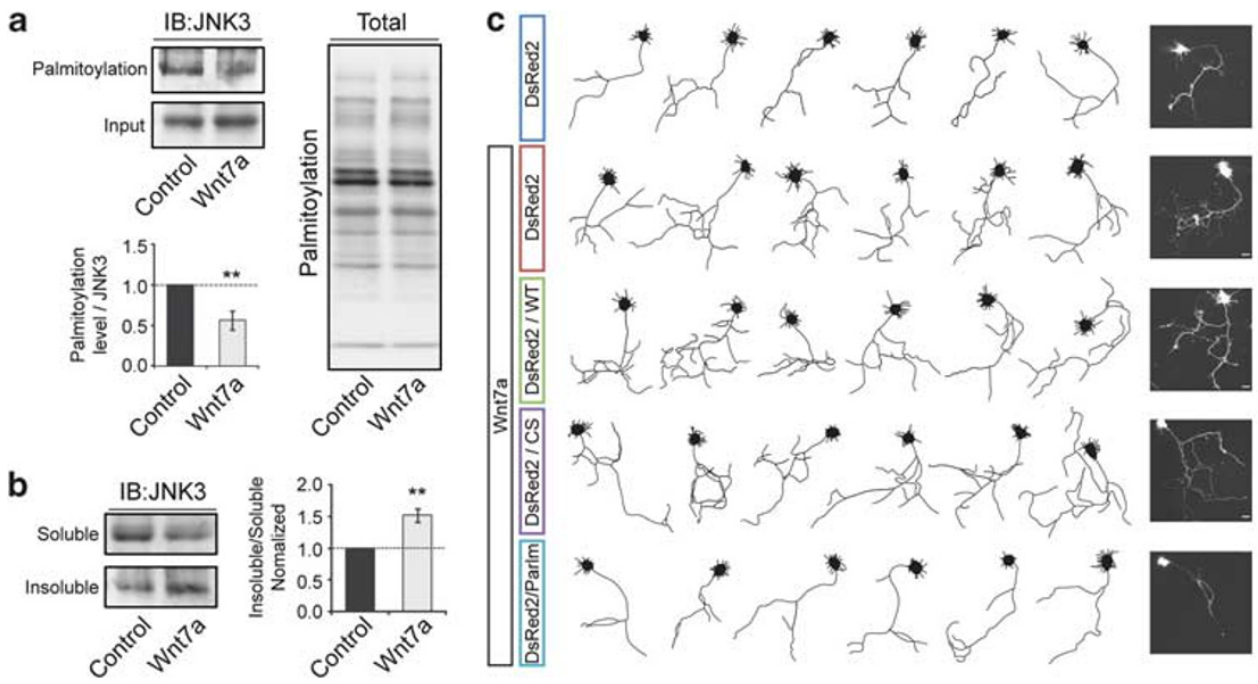

d
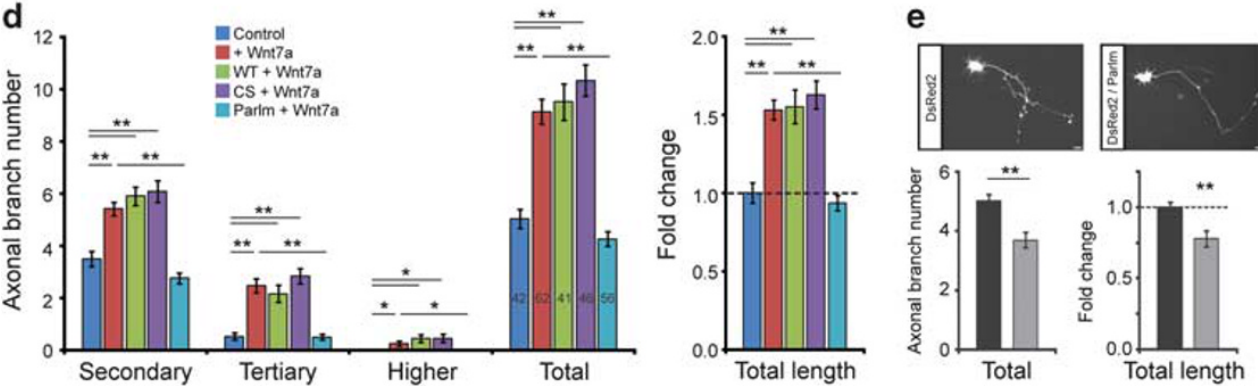

Figure 4 JNK3 palmitoylation modulates Wnt7a-regulated axonal branching. (a) JNK3 palmitoylation in neurons is regulated by Wnt7a. Brief application of Wnt7a $(200 \mathrm{ng} / \mathrm{ml}, 6 \mathrm{~h})$ to cortical neurons reduces JNK3 palmitoylation, assessed by Btn-BMCC labelling (fold change to control, Wnt7a, $0.6 \pm 0.1)$. Quantifications show mean \pm S.E.M. $t$-test. ${ }^{* *} P<0.01$. The global palmitoylation status in cortical neurons is not changed. (b) Translocation of JNK3 to the Triton-insoluble fraction is regulated by Wnt7a. Wnt7a increases the presence of JNK3 in the insoluble fraction in cortical neurons (fold change to control, Wnt7a, $1.5 \pm 0.1$ ). (c) Wnt7a-induced axon branching is saturated by JNK3 CS and blocked by pseudo-palmitoylated JNK3 (Parlm). Representative Wnt7a non-treated or treated hippocampal neurons transfected with indicated constructs are shown. Scale bar: $10 \mu \mathrm{m}$. (d) Differential effects of JNK3 CS and JNK3 Parlm on axonal branching of hippocampal neurons induced by Wnt7a. After Wnt7a induction, DsRed2 alone, JNK3 WT and JNK3 CS groups show similar numbers of total axonal branches (DsRed2 alone + Wnt7a/red, 9.1 $\pm 0.5, n=62 ;$ WT + Wnt7a/green, $9.5 \pm 0.7, n=41 ; \mathrm{CS}+$ Wnt7a/purple, $10.3 \pm 0.6, n=46 ; \mathrm{F}=1.3, P=0.28$ ) and total axonal length (fold change to DsRed2 alone, DsRed2 alone + Wnt7a, $1.5 \pm 0.4 ; \mathrm{WT}$, $1.6 \pm 0.8 ; \mathrm{CS}, 1.6 \pm 0.6 ; \mathrm{F}=0.34, P=0.71)$. The JNK3 Parlm group shows fewer axonal branches $(4.3 \pm 0.3, n=56 ; F=39.78, P<0.01)$ and reduced axonal length (fold change, $0.9 \pm 0.4 ; F=19.14, P<0.01$ ) compared with other Wnt7a-treated groups. Quantifications show mean \pm S.E.M. One-way ANOVA test. ${ }^{*} P<0.05$ and ${ }^{\star *} P<0.01$, respectively. (e) Pseudo-palmitoylated JNK3 inhibits normal axonal development. Hippocampal neurons transfected with JNK3 Parlm develop fewer axonal branches (DsRed2 alone/black, $5.0 \pm 0.2$; Parlm/gray, $3.7 \pm 0.3, n=33$ ) and shorter axons (fold change to DsRed2 alone, Parlm, $0.8 \pm 0.1$ ). $t$-test. Scale bar: $10 \mu \mathrm{m}$

involved in different signalling pathways, mechanisms with isoform selectivity would be advantageous to precisely regulate JNK activity and to mobilize JNK isoforms to the appropriate cellular compartments. Protein palmitoylation may be one candidate mechanism. In neurons, palmitoylation has multiple effects on protein trafficking, lipid raft association and other protein functions. ${ }^{20}$ Despite the prominent role played by JNK3 in the stress response, loss of palmitoylation neither affects JNK3 phosphorylation in response to stress nor changes its distribution in the nucleus and mitochondria, where JNK3 activates apoptosis pathways (Supplementary Figure S3 and data not shown). Thus, JNK3 palmitoylation may not directly participate in regulating the JNK3-mediated stress response. Our data further suggest that palmitoylation is not involved in controlling lipid raft association of JNK3 (Figure 3a). In fact, lipid raft association of unpalmitoylated JNK2 p54 and JNK1 p46 are normal. To our surprise, palmitoylation-deficient JNK3 CS shows an increase of translocation to the actin cytoskeleton, whereas promoting JNK3 palmitoylation by PAT zD15 reduces the translocation (Figure $3 e$ ). This suggests a role of palmitoylation in regulating JNK3's association with the actin cytoskeleton. Whether palmitoylation of JNK3 also regulates its distribution on microtubules requires further investigation. Several proteins that bind to and modulate the actin cytoskeleton have been identified as JNK substrates, such as paxillin. ${ }^{40}$ Our results provide a potential link between JNK3 palmitoylation and cytoskeleton modulation, but future research is needed to elucidate the underlying mechanisms.

Regulation of the neuronal cytoskeleton is essential for axonal development. ${ }^{26,30}$ In agreement with the potential role of JNK3 palmitoylation in actin cytoskeleton modulation, overexpression of palmitoylation-deficient JNK3 CS in hippocampal neurons results in more elaborate axonal branches (Figure 2). Our results showing that filopodial motility, an actin-based process, ${ }^{26}$ but not the density of filopodia is 
promoted by JNK3 CS suggests the possibility that branch extension and retraction may have a role in the JNK3 CS-induced enhancement of branching. Further studies are needed to fully understand the molecular mechanisms. The rapid kinetics of JNK3 palmitoylation provides the possibility that JNK3 regulates these highly dynamic processes (Figure 1b). Members of the Wnt family of proteins represent one group of prominent regulators of neurite development, which directly regulate cytoskeleton networks by recruiting JNK in the noncanonical Wnt pathway. ${ }^{11,14-16}$ The expressions of two Wnt members, Wnt7a and b, are substantial in hippocampal formation. ${ }^{31}$ Previous studies have found that Wnt7a regulates axonal branching and remodelling, ${ }^{15}$ whereas Wnt7b modulates the formation of complex dendritic arborization. ${ }^{14}$ In response to Wnt7a signals, a decrease of endogenous JNK3 palmitoylation and an increase of JNK3 translocation to the actin-cytoskeleton fraction was observed (Figures $4 a$ and $b$ ), suggesting that depalmitoylation of JNK3 may participate in the Wnt7a signalling pathway for axonal development regulation. This is supported by our findings showing that JNK3 CS mimics and stimulates the effects of Wnt7a on axonal branching (Figure 4). In this condition, overexpressing JNK3 CS may have hijacked endogenous pathways that are normally regulated by Wnt7a-induced JNK3 depalmitoylation. To test this hypothesis, it would be ideal to have a JNK3 that is irreversibly palmitoylated, as it may resist Wnt7a-induced depalmitoylation and thus stop downstream signalling pathways. A COOH-terminal motif of paralemmin which is irreversibly prenylated and dual palmitoylated has been shown to mimic constant palmitoylation on proteins. ${ }^{35}$ This pseudo-palmitoylation is able to restore intracellular trafficking of the cytosol protein PSD-95 even in the presence of the palmitoylation inhibitor 2-BrPA. ${ }^{34,36}$ Overexpression of pseudo-palmitoylated JNK3 Parlm was able to completely abolish the effects of Wnt7a, indicating the involvement of JNK3 palmitoylation in Wnt7a signalling pathways for axonal development. Interestingly, Jnk3 ${ }^{-1-}$ mice $^{4,5}$ and neurons overexpressing JNK3 WT (Figure 2) show normal axonal development. Only manipulations of JNK3 palmitoylation produce abnormal branching phenotypes (Figures $2 \mathrm{~b}, \mathrm{c}$ and $4 \mathrm{e}$ ). Given that JNK1 and JNK2 have been shown to play a role in axonal development, it is thus possible that JNK pathways regulating axonal development are redundant. It is also possible that JNK isoforms may function differently under certain conditions. Our results suggest that JNK3 is not a constitutive mediator of axonal development, but may act as an important modulator of axonal development in response to upstream signals by palmitoylation. In sum, our findings reveal a novel isoform-selective mechanism underlying JNK regulation, and suggest a potential role of JNK3 in axonal branching.

\section{Materials and Methods}

Cell cultures and materials. All animal experiments were approved by the Animal Care Committee of the University of British Columbia. Primary cortical or hippocampal neuronal cultures were prepared from Wistar rats (UBC Animal Care Centre, Vancouver, BC, Canada) at embryonic days 18-19. Cultures were maintained in Neurobasal medium supplemented with B27 and $0.5 \mathrm{mM}$ glutamax (Invitrogen, Carlsbad, CA, USA) as described previously.2 ${ }^{22}$ For biochemical studies, cortical neurons were seeded in six-well culture plates with a density of $7.5 \times 10^{5}$ cells/well. Neurons of 5 or 14-17 DIV were used for experiments. For immunostaining, hippocampal neurons were seeded on glass coverslips in 12well plates with a density of $1.3 \times 10^{5}$ cells/well. To assess axonal development, hippocampal neurons at 5 DIV were transfected with pDsRed2 plus other indicated constructs at 3:1 ratio with Lippofectamine2000 (Invitrogen). Recombinant Wnt7a protein (R \& D Systems, Minneapolis, MN, USA) was added at 5 DIV for $48 \mathrm{~h}$. At 7 DIV, neurons were fixed, stained and analyzed. HEK293 and Cos7 cells were maintained in DMEM (Invitrogen) supplemented with 10\% fetal bovine serum and $1 \%$ penicillin/streptomycin. Cells were transfected with Calcium Phosphate Transfection reagent (Promega, San Luis Obispo, CA, USA) or Lippofectamine2000 and used 16-24h later as described previously. The actin depolymerizers Cyt.D $(1 \mu \mathrm{M})$ and Lat.A $(1 \mu \mathrm{M})$ were obtained from Calbiochem (San Diego, CA, USA). [9,10- $\left.{ }^{3} \mathrm{H}\right]$ palmitic acid (57 Ci/mmol) and biotin (Btn)-BMCC were purchased from PerkinEImer Life Sciences (Waltham, MA, USA). Optiprep was purchased from Axis Shield (Dundee, Scotland). Other assay reagents without indication were obtained from Sigma (St. Louis, MO, USA)

Plasmids and antibodies. The CDNA of EGFP-tagged JNK3 (GFP-JNK3) was subcloned into $\mathrm{pcDNA3}$, which served as the template for generating point mutations on JNK3 (JNK3 1CS, JNK3 2CS, JNK3 CS) with PCR. To create the JNK3 Parlm construct, 12 amino acids at the end of the $\mathrm{COOH}$-terminus of the protein paralemmin ${ }^{34-36}$ were inserted at the $\mathrm{COOH}$-terminus of the pcDNA3 GFP-JNK3 CS construct. GFP-JNK1 and GFP-JNK2 expression constructs were created by cloning CDNA sequences into pEGFP-C1 and subcloned into the pcDNA3 vector. A PAT activity-deficient $\mathrm{zD} 15$ was created by point mutation of the Cys residue within the DHHC domain to Ser with PCR, and cloned into pcDNA3 to generate the expression construct. The vectors encoding myc-zD15, myc-zD20 and myc-zD23 were generously provided by Dr. Alaa El-Husseini. The GFP-JNK3 construct was a gift from Dr. Vsevolod V Gurevich. The primary antibodies used to detect JNK1, $\beta$-actin and caveolin-1 were obtained from Abcam (Cambridge, MA, USA). Anti-GFP was purchased from Sigma. Anti-Tau-1 was purchased from Millipore (Billerica, MA, USA). Purified IgG was supplied by Jackson ImmunoResearch (Baltimore, MD, USA). All other primary antibodies were purchased from Cell Signaling Technology (Danvers, MA, USA). Secondary antibodies were HRP-conjugated anti-mouse (Cell Signaling Technology), antirabbit (PerkinElmer), anti-GFP (Santa Cruz Biotechnology, Santa Cruz, CA, USA), anti-myc (Invitrogen) and avidin (GE Healthcare, Piscataway, NJ, USA). Texas red (TR)-phalloidin, fluorescent Alexa 488- and Alexa 568-conjugated anti-mouse lgG1, IgG2a and anti-rabbit IgG were obtained from Invitrogen.

${ }^{3} \mathrm{H}$-palmitate metabolic labelling and Btn-BMCC labelling. To label palmitoylated proteins, 14 DIV neuronal cultures were incubated in Neurobasal medium containing $0.8 \mathrm{mCi} / \mathrm{ml}^{3} \mathrm{H}$-palmitate for $3 \mathrm{~h}$. 2-BrPA $(100 \mu \mathrm{M})$ was added to the medium $2 \mathrm{~h}$ before labelling to inhibit palmitoylation. ${ }^{22}$ For pulse-chase experiments, neuronal cultures were first labelled with ${ }^{3} \mathrm{H}$-palmitate for $3 \mathrm{~h}$, and then incubated for 4 or $6 \mathrm{~h}$ in conditioned Neurobasal medium supplemented with unlabelled palmitate $(100 \mu \mathrm{M})$. JNK3 was then immunoprecipitated and incubated with or without $1 \mathrm{M} \mathrm{HAM}$ for $1 \mathrm{~h}$ at RT before analysis with SDS-PAGE. Btn-BMCC labelling was conducted as previously described. ${ }^{22}$ Briefly, after incubating with $\mathrm{N}$-ethylmaleimide to block free sulfhydryl groups on proteins, samples were either immunoprecipitated with anti-GFP or cleaned with Micro Bio-Spin6 Columns (Bio-Rad, Hercules, CA, USA). Purified precipitates or samples were treated with or without $1 \mathrm{M}$ HAM and $1 \mu \mathrm{M}$ Btn-BMCC (Pierce, Rockford, IL, USA) to label palmitoylation sites. The presence of Btn on JNK proteins was then analyzed by SDS-PAGE with HRP-conjugated anti-avidin.

Protein extraction and immunoblotting. The protocols for protein extraction have been described previously. ${ }^{22}$ For immunoprecipitation, cultured cells were washed with ice-cold PBS, followed by incubation with $0.5 \mathrm{ml}$ lysis buffer containing $50 \mathrm{mM}$ Tris- $\mathrm{HCl}$ (pH 7.4), $1 \mathrm{mM}$ EDTA, $1 \mathrm{mM}$ EGTA, $150 \mathrm{mM} \mathrm{NaCl}, 1 \%$ Triton X-100, 0.1\% SDS supplemented with Complete Protease Inhibitor Tablets (Roche Applied Science, Indianapolis, IN, USA) and $1 \mathrm{mM}$ PMSF. Lysates were precleaned by incubating with protein A-Sepharose beads (Roche Applied Science) for $1 \mathrm{~h}$ at $4^{\circ} \mathrm{C}$, followed by incubation with indicated antibodies $\left(>16 \mathrm{~h}\right.$ at $4^{\circ} \mathrm{C}$ ) and protein A-Sepharose bead $\left(2 \mathrm{~h}\right.$ at $\left.4^{\circ} \mathrm{C}\right)$. Immunoprecipitates were washed three times with lysis buffer, boiled in $2 \times$ loading buffer with $1 \mathrm{mM}$ dithiothreitol for $3 \mathrm{~min}$, and analyzed with SDS-PAGE. For autoradiography, protein samples were separated by $8 \%$ SDS-PAGE and incubated with Amplify reagent (Amersham 
Biosciences, Sydney, NSW, Australia). Gels were dried under vacuum and exposed to Hyperfilm (Amersham Biosciences) with intensifying screens at $-80^{\circ} \mathrm{C}$ for $30-50$ days. The relative intensities of the bands on autoradiograms and western blots were analyzed and quantified using Image J (NIH).

Immunostaining and laser microscopy. Cultured hippocampal neurons on glass coverslips were used for immunocytochemistry and time-lapse studies. Immunostaining was conducted as described previously.22 For staining of the actin cytoskeleton, coverslips were incubated with TR-phalloidin in PBS for $1 \mathrm{~h}$ at RT. To assess axonal development, cultured hippocampal neurons were stained with antiTau-1 antibody. Coverslips were mounted with ProLong Gold Antifade Reagent containing DAPI (Invitrogen). Fluorescence was captured with a $\times 60$ objective affixed to an Olympus Fluoview 2000 confocal microscope (Olympus, Richmond Hill, ON, Canada) or using a Zeiss Axiovert 200 microscope (Zeiss, Toronto, ON, Canada). For time-lapse imaging, 6 DIV neurons transfected with the indicated constructs were mounted on a home-made chamber filled with Neurobasal medium (minus phenol red) and supplements at $30^{\circ} \mathrm{C}$. Images were captured at every $5 \mathrm{~s}$ over $150 \mathrm{~s}$ using a $\times 60$ oil immersion lens on a Zeiss Axio Observer Z1 microscope. All images were analyzed with ImageJ and processed using Adobe Photoshop.

Morphometric analysis. The tip number and length of axonal branches were quantified using ImageJ with NeuronJ add-on, after tracing axonal branches. The primary axon is defined as the longest continuous path from the somata. The secondary axons are defined as branches initiated from the primary axon, and so on. The terminal points of all axonal branches are defined as axon tips. To illustrate the motility of axonal filopodia, the differences in pairs of successive frames from 30 time-lapse images were extracted by ImageJ arithmetically. ${ }^{26}$ The resulting 29 images were added up to generate a final image showing all the differences. A greater number of pixels represents higher motility. To quantify filopodia motility, the $\mathrm{MI}$, which measures the areas that a filopodium occupies over time, was used as described previously. ${ }^{26}$ Briefly, images were adjusted using a threshold throughout the entire time-lapse stack and then binarized. A total of 10 filopodia from each neuron were randomly chosen for further analysis. The areas that a filopodium occupied throughout the stack were measured and calculated using the formula: $\mathrm{Ml}=[\operatorname{area}(\max )-\operatorname{area}(\min )] \star 2 /[\operatorname{area}(\max )+\operatorname{area}(\min )]$.

Cell fractionation. For isolation of the Triton-insoluble fraction, cells were washed with cold PBS and lysed with lysis buffer with $1 \%$ Triton X-100 for 5 min at RT. Cells were then scraped into a tube and homogenized by pipetting with a 200- $\mu$ l tip. After centrifugation for $10 \mathrm{~min}$ at $13000 \mathrm{~g}$ at $4^{\circ} \mathrm{C}$, the pellet was resolved with $1 \times$ SDS loading buffer and was defined as the cytoskeleton fraction. The same volume of samples from the supernatant and pellet fractions was then subjected to SDS-PAGE analysis.

Statistics. All values in text and figures are presented as mean \pm standard error of the mean (S.E.M.). Student's t-test or one-way ANOVA test was performed using Excel software (Microsoft). The limit of statistical significance was set at a $P$-value $<0.05$.

\section{Conflict of Interest}

The authors declare no conflict of interest.

Acknowledgements. We thank Dr. Vsevolod V Gurevich for providing the GFP-JNK3 construct. We also thank Dr. Alaa El-Husseini for providing DNA constructs. We thank Dr. Ann Marie Craig for technique help and critical reading of the manuscript. We also thank members of Dr. Cynader's lab for helpful suggestions. This work was supported by grants from the CIHR (MSC) and Natural Sciences and Engineering Research Council of Canada (NSERC) (MSC).

1. Bogoyevitch MA. The isoform-specific functions of the c-Jun N-terminal kinases (JNKs): differences revealed by gene targeting. Bioessays 2006; 28: 923-934.

2. Haeusgen W, Herdegen T, Waetzig V. The bottleneck of JNK signaling: molecular and functional characteristics of MKK4 and MKK7. Eur J Cell Biol 2011; 90: 536-544.
3. Yang DD, Kuan CY, Whitmarsh AJ, Rincon M, Zheng TS, Davis RJ et al. Absence of excitotoxicity-induced apoptosis in the hippocampus of mice lacking the Jnk3 gene. Nature 1997; 389: 865-870.

4. Hunot S, Vila M, Teismann P, Davis RJ, Hirsch EC, Przedborski S et al. JNK-mediated induction of cyclooxygenase 2 is required for neurodegeneration in a mouse model of Parkinson's disease. Proc Natl Acad Sci U S A 2004; 101: 665-670.

5. Kuan CY, Whitmarsh AJ, Yang DD, Liao G, Schloemer AJ, Dong $C$ et al. A critical role of neural-specific JNK3 for ischemic apoptosis. Proc Natl Acad Sci USA 2003; 100: 15184-15189.

6. Brecht S, Kirchhof R, Chromik A, Willesen M, Nicolaus T, Raivich G et al. Specific pathophysiological functions of JNK isoforms in the brain. Eur J Neurosci 2005; 21: 363-377.

7. Chang L, Jones $Y$, Ellisman MH, Goldstein LS, Karin M. JNK1 is required for maintenance of neuronal microtubules and controls phosphorylation of microtubule-associated proteins. Dev Cell 2003; 4: 521-533.

8. Tararuk T, Ostman N, Li W, Bjorkblom B, Padzik A, Zdrojewska J et al. JNK1 phosphorylation of SCG10 determines microtubule dynamics and axodendritic length. J Cell Biol 2006; 173: 265-277.

9. Bjorkblom B, Ostman N, Hongisto V, Komarovski V, Filen JJ, Nyman TA et al. Constitutively active cytoplasmic c-Jun $\mathrm{N}$-terminal kinase 1 is a dominant regulator of dendritic architecture: role of microtubule-associated protein 2 as an effector. $J$ Neurosci 2005; 25: 6350-6361.

10. Waetzig V, Herdegen T. A single c-Jun N-terminal kinase isoform (JNK3-p54) is an effector in both neuronal differentiation and cell death. J Biol Chem 2003; 278: 567-572.

11. Ciani L, Salinas PC. c-Jun N-terminal kinase (JNK) cooperates with Gsk3beta to regulate Dishevelled-mediated microtubule stability. BMC Cell Biol 2007; 8: 27.

12. Kimberly WT, Zheng JB, Town T, Flavell RA, Selkoe DJ. Physiological regulation of the beta-amyloid precursor protein signaling domain by c-Jun $\mathrm{N}$-terminal kinase JNK3 during neuronal differentiation. J Neurosci 2005; 25: 5533-5543.

13. Oliva Jr AA, Atkins CM, Copenagle L, Banker GA. Activated c-Jun N-terminal kinase is required for axon formation. J Neurosci 2006; 26: 9462-9470.

14. Rosso SB, Sussman D, Wynshaw-Boris A, Salinas PC. Wnt signaling through Dishevelled, Rac and JNK regulates dendritic development. Nat Neurosci 2005; 8: 34-42.

15. Lucas FR, Salinas PC. WNT-7a induces axonal remodeling and increases synapsin I levels in cerebellar neurons. Dev Biol 1997; 192: 31-44.

16. Salinas PC, Zou Y. Wnt signaling in neural circuit assembly. Annu Rev Neurosci 2008; 31: 339-358.

17. Bogoyevitch MA, Kobe B. Uses for JNK: the many and varied substrates of the c-Jun N-terminal kinases. Microbiol Mol Biol Rev 2006; 70: 1061-1095.

18. Kuan CY, Yang DD, Samanta Roy DR, Davis RJ, Rakic P, Flavell RA. The Jnk1 and Jnk2 protein kinases are required for regional specific apoptosis during early brain development. Neuron 1999; 22: 667-676.

19. Park HS, Huh SH, Kim MS, Lee SH, Choi EJ. Nitric oxide negatively regulates c-Jun $\mathrm{N}$-terminal kinase/stress-activated protein kinase by means of S-nitrosylation. Proc Natl Acad Sci U S A 2000; 97: 14382-14387.

20. El-Husseini Ael D, Bredt DS. Protein palmitoylation: a regulator of neuronal development and function. Nat Rev Neurosci 2002; 3: 791-802.

21. Biilmakers MJ, Marsh M. The on-off story of protein palmitoylation. Trends Cell Biol 2003; 13: $32-42$.

22. Yang G, Xiong W, Kojic L, Cynader MS. Subunit-selective palmitoylation regulates the intracellular trafficking of AMPA receptor. Eur J Neurosci 2009; 30: 35-46.

23. Yamazaki M, Fukaya M, Abe M, Ikeno K, Kakizaki T, Watanabe M et al. Differential palmitoylation of two mouse glutamate receptor interacting protein 1 forms with different N-terminal sequences. Neurosci Lett 2001; 304: 81-84.

24. Guo C, Whitmarsh AJ. The beta-arrestin-2 scaffold protein promotes c-Jun N-terminal kinase-3 activation by binding to its nonconserved $\mathrm{N}$ terminus. J Biol Chem 2008; 283: 15903-15911.

25. Bodmer D, Levine-Wilkinson S, Richmond A, Hirsh S, Kuruvilla R. Wnt5a mediates nerve growth factor-dependent axonal branching and growth in developing sympathetic neurons. J Neurosci 2009; 29: 7569-7581.

26. Chang S, De Camilli P. Glutamate regulates actin-based motility in axonal filopodia. Nat Neurosci 2001; 4: 787-793.

27. Nieto-Miguel T, Gajate C, Gonzalez-Camacho F, Mollinedo F. Proapoptotic role of Hsp90 by its interaction with $\mathrm{C}$-Jun $\mathrm{N}$-terminal kinase in lipid rafts in edelfosine-mediated antileukemic therapy. Oncogene 2008; 27: 1779-1787.

28. Vetrivel KS, Cheng H, Lin W, Sakurai T, Li T, Nukina $N$ et al. Association of gammasecretase with lipid rafts in post-Golgi and endosome membranes. J Biol Chem 2004; 279 : 44945-44954.

29. Bodin S, Soulet C, Tronchere H, Sie P, Gachet C, Plantavid M et al. Integrin-dependent interaction of lipid rafts with the actin cytoskeleton in activated human platelets. J Cell Sci 2005; 118 (Pt 4): 759-769.

30. Luo L. Actin cytoskeleton regulation in neuronal morphogenesis and structural plasticity. Annu Rev Cell Dev Biol 2002; 18: 601-635.

31. Gogolla N, Galimberti I, Deguchi Y, Caroni P. Wnt signaling mediates experience-related regulation of synapse numbers and mossy fiber connectivities in the adult hippocampus. Neuron 2009; 62: 510-525. 
32. Drisdel RC, Green WN. Labeling and quantifying sites of protein palmitoylation. Biotechniques 2004; 36: 276-285.

33. Davis EK, Zou Y, Ghosh A. Wnts acting through canonical and noncanonical signaling pathways exert opposite effects on hippocampal synapse formation. Neural Dev 2008; 3: 32

34. El-Husseini Ael D, Schnell E, Dakoji S, Sweeney N, Zhou Q, Prange O et al. Synaptic strength regulated by palmitate cycling on PSD-95. Cell 2002; 108: 849-863.

35. Kutzleb C, Sanders G, Yamamoto R, Wang X, Lichte B, Petrasch-Parwez E et al. Paralemmin, a prenyl-palmitoyl-anchored phosphoprotein abundant in neurons and implicated in plasma membrane dynamics and cell process formation. J Cell Biol 1998; 143: $795-813$.
36. El-Husseini AE, Craven SE, Chetkovich DM, Firestein BL, Schnell E, Aoki C et al. Dual palmitoylation of PSD-95 mediates its vesiculotubular sorting, postsynaptic targeting, and ion channel clustering. J Cell Biol 2000; 148: 159-172.

37. Kyriakis JM, Banerjee P, Nikolakaki E, Dai T, Rubie EA, Ahmad MF et al. The stressactivated protein kinase subfamily of c-Jun kinases. Nature 1994; 369: 156-160

38. Zhao $Y$, Herdegen $T$. Cerebral ischemia provokes a profound exchange of activated JNK isoforms in brain mitochondria. Mol Cell Neurosci 2009; 41: 186-195.

39. Amura CR, Marek L, Winn RA, Heasley LE. Inhibited neurogenesis in JNK1-deficient embryonic stem cells. Mol Cell Biol 2005; 25: 10791-10802.

40. Huang C, Rajfur Z, Borchers C, Schaller MD, Jacobson K. JNK phosphorylates paxillin and regulates cell migration. Nature 2003; 424: 219-223.

Supplementary Information accompanies the paper on Cell Death and Differentiation website (http://www.nature.com/cdd) 